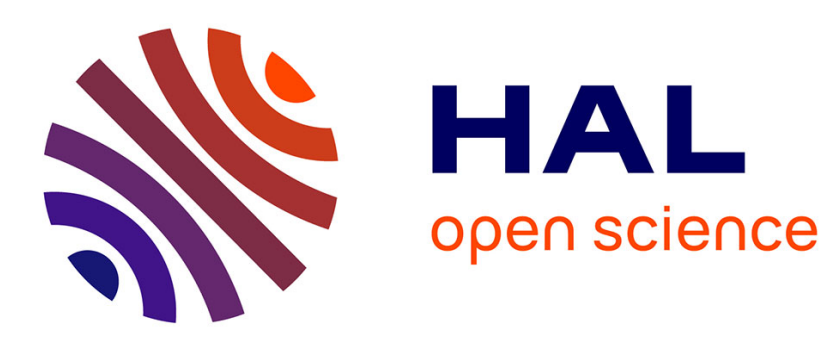

\title{
Gibbs Sampling Based Distributed OFDMA Resource Allocation
}

Virgile Garcia, Chung Shue Chen, Yiqing Zhou, Jinglin Shi

\section{To cite this version:}

Virgile Garcia, Chung Shue Chen, Yiqing Zhou, Jinglin Shi. Gibbs Sampling Based Distributed OFDMA Resource Allocation. Science China Information Sciences, 2014. hal-00927286

\section{HAL Id: hal-00927286 \\ https://hal.inria.fr/hal-00927286}

Submitted on 12 Jan 2014

HAL is a multi-disciplinary open access archive for the deposit and dissemination of scientific research documents, whether they are published or not. The documents may come from teaching and research institutions in France or abroad, or from public or private research centers.
L'archive ouverte pluridisciplinaire HAL, est destinée au dépôt et à la diffusion de documents scientifiques de niveau recherche, publiés ou non, émanant des établissements d'enseignement et de recherche français ou étrangers, des laboratoires publics ou privés. 


\title{
Gibbs Sampling Based Distributed OFDMA Resource Allocation
}

\author{
Virgile Garcia ${ }^{* \dagger}$, Chung Shue Chen ${ }^{\ddagger}$, Yiqing Zhou (corresponding)*†, Jinglin Shi*† \\ *Wireless Technology Research Center, Institute of Computing Technology, Chinese Academy of Science, China \\ ${ }^{\dagger}$ Beijing Key Laboratory of Mobile Computing and Pervasive Device \\ ${ }^{\ddagger}$ Alcatel-Lucent Bell Labs, Centre de Villarceaux, 91620 Nozay, France \\ Emails: \{virgilegarcia, zhouyiqing, sjl\}@ict.ac.cn, cs.chen@alcatel-lucent.com
}

\begin{abstract}
In this paper, we present a distributed resource and power allocation scheme for multiple-resource wireless cellular networks. The global optimization of multi-cell multi-link resource allocation problem is known NP-hard in the general case. We use Gibbs sampling based algorithms to perform a distributed optimization that would lead to the global optimum of the problem. The objective of this paper is to show how to use the Gibbs sampling (GS) algorithm and its variant the Metropolis-Hasting (MH) algorithm. We also propose an enhanced method of the MH algorithm, based on a priori-known target state distribution, which improves the convergence speed without increasing the complexity. Furthermore, we study different temperature cooling strategies and investigate their impact on the network optimization and convergence speed. Simulation results have also shown the effectiveness of the proposed methods.
\end{abstract}

\section{Index Terms}

Radio resource allocation, power control, interference management, distributed optimization, Gibbs sampling, MetropolisHasting.

\section{INTRODUCTION}

Cellular networks are facing an exponential increase of capacity demand and a key solution to overcome this problem is to multiply the number of access points or base stations (BSs) in an effective way [1]. However, the multiplication of BSs, and in particular when a frequency reuse factor of one is used, would yield substantial inter-cell interference and lead to an interference-limited network.

Radio resource management and interference coordination have been extensively studied in the literature [2], [3], trying to provide optimal or near-optimal schemes. In practice, this class of multi-cell or multi-link resource allocation optimization problems is generally non-convex and very hard to solve, especially in a network with a large number of BSs.

Various stochastic optimization methods have been introducedsuch as particle swarm optimization, genetic algorithm, ant colony or tabu search [4]. These approaches generally require a knowledge of the system, and some need several instances of the system, which are only feasible in an off-line, centralized approach. Different to these complex centralized schemes designed to find a global optimal solution, distributed algorithms have drawn more and more attentions recently due to the low complexity and low requirement on backhauling and processing capability. Most of the time, sub-optimal solutions are provided by distributed algorithms, such as those using game theory framework [5], often face the issue of converging to equilibria (e.g., Nash equilibrium) that are sub-optimal solutions.

Gibbs sampling (GS) algorithm [6] is stochastic optimization method that can converge to the global optimal solution of non-convex problem. Under some conditions, it can even be applied in a fully distributed manner, with only local decisions. The Metropolis-Hasting (MH) algorithm [7] is also a widely used MCMC method and has same methodology, which however has a lower complexity but a slower convergence rate.

The first application of GS for wireless networks aimed to minimize co-channel interference and offer optimal frequency band allocation in WiFi networks [8]. More recently, the technique has been successfully extended to the user association and power control problem [9] and the transmit precoding and power efficiency problem [10] for cellular networks. However, these works are limited by the use of a single resource for the optimization problem. In [11], the Gibbs sampling method is generalized for any arbitrary utility function, by designing an extended interference graph with respect to cellular networks.

Although theoretical backgrounds of the Gibbs sampling algorithm are well known, its application to radio resource allocation is relatively limited so far and previous works only focused on the classical very slow temperature ${ }^{1}$ cooling and using only one specific algorithm (either GS or MH).

In this paper, considering a large cellular OFDMA network with multiple resources, we apply GS and MH methods for the resource and power allocation optimization and conduct a comparative study. Also, we investigate the performance of different temperature cooling strategies to improve the convergence speed of the classical Gibbs sampling. Moreover, given

\footnotetext{
${ }^{1}$ Note that in GS and MH algorithms, a key parameter called temperature will tune the state sampling and transition and impact the speed and optimality of the convergence.
} 
a prior statistical knowledge of the solution, we propose an enhanced MH algorithm, namely "guided" MH, to speed up the MH convergence while keeping a low complexity. Simulations are carried out to show the performance of the GS, MH and guided-MH algorithms, compared to a deterministic best response (BR) method.

We show that although the logarithmic cooling is optimal in theory, fast cooling strategies such as exponential cooling can be more suitable for the resource and power allocation problem in practice. Also, we show that the guided-MH algorithm allows a faster convergence compared to the classical $\mathrm{MH}$ algorithm without increasing its complexity, and may have other interesting applications.

The rest of the paper is organized as follows. In Section II, the system model is presented and the power and resource allocation problem is formulated. In Section III, we develop the Gibbs sampling formulation and its variants, including the proposed guided-MH algorithm and the various temperature cooling strategies studied. In Section IV, we show how to cast the resource and power allocation problem to use the Gibbs sampling algorithms. In Section V, we provide simulation results under OFDMA multi-cell environment to compare the performance of the studied methods. Finally, Section VI contains the conclusion.

\section{System ModeL}

\section{A. OFDMA cellular network}

We consider the downlink transmission in a classical multi-cell system. Different to the work in [8]-[10] that focused on a single channel per user, we consider in this paper that a user may take multiple orthogonal resources, as in 3GPP-LTE orthogonal frequency-division multiple access (OFDMA).

The power and resource allocation is performed locally at each BS for their respective users. We consider reuse-1 system, i.e., all resource blocks (RBs) are available at each BS. Each user $i \in \mathcal{U}$ is associated to the BS $b_{i} \in \mathcal{B}$ that has the smallest path-loss, where $\mathcal{U}$ is the set of users and $\mathcal{B}$ is the set of BSs. We consider that the total bandwidth is divided into $K$ orthogonal resources, each experiencing an independent and flat Rayleigh fading.

Notice that under 3GPP-LTE, each user can be allocated any number of RBs. However without multi-user MIMO techniques, a RB cannot be allocated to more than one user within a cell. The signal-to-interference-plus-noise ratio (SINR) of a user $i \in \mathcal{U}$ on the resource $k \in[1, K]$ is thus given by:

$$
\gamma_{i, k}=\frac{P_{b_{i}, k} g_{b_{i}, i, k}}{\sum_{b_{j} \neq b_{i}} P_{b_{j}, k} g_{b_{j}, i, k}+\sigma_{i}^{2}},
$$

where $P_{b_{i}, k}$ is the transmit power of BS $b_{i}$ destined to the user $i$ on the resource $k \in \mathbb{Z}^{+}, g_{b_{i}, i, k}$ is the channel gain, including fading, between the BS $b_{i}$ and the user $i$, and $\sigma_{i}^{2}$ is the thermal noise variance, supposed equal for all users here. Let $C_{i, k}$ denote the data rate achievable by the resource $k$ for user $i$. The total data rate of a user $i$ is given by:

$$
C_{i}=\sum_{k \in[1, K]} \log _{2}\left(1+\gamma_{i, k}\right) .
$$

In this paper, we consider the Shannon capacity, yet any other metric can be employed in the following optimization.

\section{B. Resource and power allocation problem formulation}

In OFDMA based system like LTE with no multi-user MIMO, orthogonal resources are assigned to users to avoid intra-cell interference. Thus, aiming at inter-cell optimization and for simplification, a single user is focused for each cell, which can use any of the $K$ resources. However, it should be noted that the result can be easily extended to more general case.

We consider that the BSs can adapt their power level on each resource, $P_{b, k}, \forall b \in \mathcal{B}, \forall k \in[1, K]$, independently. We note $\mathbf{P}_{b}$ the vector of power allocation of $\mathrm{BS} b$, and $\mathbf{P}$ the global matrix of power allocations.

Considering the data rates of the users as the basic metric of interest, a generic optimization problem is formulated as follows:

$$
\begin{aligned}
& \min U(\mathbf{C}) ; \\
& \text { s.t. : } \\
& P_{b, k} \geq 0, \forall b \in \mathcal{B}, \forall k \in[1, K], \\
& \sum_{k \in[1, K]} P_{b, k} \leq P_{\max }, \forall b \in \mathcal{B},
\end{aligned}
$$

where $\mathbf{C} \triangleq\left[C_{1}, C_{2}, \ldots\right]$ is the vector of aggregated data rates of all the users, and $U(\cdot)$ is a chosen utility function that we will minimize. The total transmit power of a BS is limited by $P_{\max }$. With a little abuse of the name, we simply call $U(\cdot)$ the utility function instead of cost function.

A utility function can be used for different objective, e.g., maximizing system capacity, maximizing fairness, minimizing energy consumption, etc. 
It has been shown that applying a fairness utility functions does not only help to maintain resource sharing fairness but also favors a system-wide throughput maximization: the global optimization makes a tradeoff between users' selfish and altruistic behavior [10]. Meanwhile, it would also bring energy efficiency merit: transmitters are encouraged to lower their transmit power so as to avoid high interference to neighboring users. Therefore, in this paper, the potential delay fairness [12] criterion is employed as utility function:

$$
U(\mathbf{C})=\sum_{i \in \mathcal{U}} \frac{1}{C_{i}}
$$

where $1 / C_{i}$ represents the time required for the transmission of an unitary size. Indeed, the method allows any arbitrary utility function (see e.g., [11]).

\section{GiBBS SAMPLING OPTIMIZATION}

In this study, we focus on the Gibbs sampling and its variant the Metropolis-Hasting algorithm. The key idea of the GS and $\mathrm{MH}$ algorithms in brief is to converge, iteratively, to the global optimal solution by probabilistically transiting among the possible states of the system. Based on the interactions of nodes in the graph, the guided random selection of states allows the optimization to avoid getting trapped in local optimal points.

\section{A. Definitions}

To begin with, we define a graph $\mathcal{G}=(\mathcal{U}, \mathcal{V})$, where $\mathcal{U}$ is the set of nodes that represent the set of users, and $\mathcal{V}$ is the set of edges between the nodes. $\mathcal{G}$ is also associated to the following:

- An edge $(i, j)$ between two nodes $i$ and $j$ is set when either $i$ or $j$ affects the performance of the other.

- Each node $i \in \mathcal{U}$ has a state $s_{i}$ from a finite state set $\mathcal{S}_{i}$. The sets of states may be different for each node.

- The graph $\mathcal{G}$ is associated with the global energy metric $\mathcal{E}$, called global energy, which is a function of all the states of the nodes $\mathbf{s}=\left[s_{1}, s_{2}, \ldots\right]$ and will be minimized in the optimization.

Note that $\mathcal{G}$ is a Gibbs random field if $\mathbf{s}$ follows the distribution $\pi(\mathbf{s})=\kappa \exp (-\mathcal{E}(\mathbf{s}) / T)$, where $\kappa$ is a normalization factor. $T$ is an important parameter called temperature that scales the probability distribution of the system. It can be a fixed constant or be varied with the time. In the latter case, we say annealing.

A centralized processing unit can perform a global sampling and optimization of the problem; however, distributed solution are much preferred. To be able to distribute the GS and $\mathrm{MH}$ algorithms, the global energy has to be represented in a certain structural setup [13]: the global energy $\mathcal{E}$ has to be derivable from a sum of potential functions, over all the cliques of the graph such that

$$
\mathcal{E}(\mathbf{s})=\sum_{c \in \mathcal{C}} V(c, \mathbf{s}),
$$

where $\mathcal{C}$ is the set of all cliques, and $V$ is the potential function, which represents the contribution of $c$ to the global energy, taking into account users' mutual constraints (conflict and interaction).

Based on the above notion, we define the local energy $\mathcal{E}_{i}, \forall i \in \mathcal{U}$, as follows:

$$
\mathcal{E}_{i}(\mathbf{s})=\sum_{c \in \mathcal{C} \mid i \in c} V(c, \mathbf{s}) .
$$

The local energy function of a node $i$ only depends on the states and interactions of nodes in a neighborhood around $i$, i.e., it does not require a system global knowledge. Performing an iterative stochastic minimization of the local energies, this optimization would lead to the global minimum of the global energy.

\section{B. Classical sampling methods}

The basic idea of the sampling process is as follows: iteratively, each node updates its state based on the current evaluation of the decoupled system energy and on the impact of the new (to be chosen) state. The selection of the new state depends on the algorithm used:

1) Gibbs sampler algorithm: The GS algorithm [6] consists of, when the node $i$ updates its state, evaluating all the possible states $s \in \mathcal{S}_{i}$ and select a new state following a probability distribution. First, we compute the local energy $\mathcal{E}_{i}(s), \forall s \in \mathcal{S}_{i}$. Secondly, we define the probability distribution based on the local energy of each state. The probability that the state $s_{i} \in \mathcal{S}_{i}$ will be chosen is given below:

$$
p_{i}\left(s_{i}\right)=\frac{\exp \left(-\mathcal{E}_{i}\left(s_{i}\right) / T\right)}{\sum_{s \in \mathcal{S}_{i}} \exp \left(-\mathcal{E}_{i}(s) / T\right)} .
$$

Thirdly, we choose a new state (configuration) to be applied to $i$ based on the Gibbs probability distribution (10).

This method requires the computation of the local energy for all $s_{i} \in \mathcal{S}_{i}$ of a node. Note that the optimization therefore should apply on a reasonable number (size) of possible states. 
2) Metropolis-Hasting algorithm: The main difference between the GS method and the MH method is that the latter will only consider a single candidate state during the update process. Consider a node $i \in \mathcal{U}$, and assume that it is currently associated with a state $s_{1} \in \mathcal{S}_{i}$. When it updates its state, it will randomly choose a candidate state $s_{2} \in \mathcal{S}_{i}$ and evaluate its performance. The node $i$ will then select the new state $s_{2}$ over $s_{1}$ with the following probability:

$$
p_{i}\left(s_{2}\right)=\min \left(1, \exp \left(\frac{\mathcal{E}_{i}\left(s_{1}\right)-\mathcal{E}_{i}\left(s_{2}\right)}{T}\right)\right) .
$$

In other words, if the candidate state has a lower local energy, it will always be chosen. If its local energy is higher, it will be chosen with a probability, given by (11).

The complexity and computation cost of the MH compared to the GS algorithm is divided by the number of possible states for the node, due to the single candidate evaluation of the $\mathrm{MH}$ algorithm.However, the convergence time of $\mathrm{MH}$ algorithm can also be longer, due to a slower sampling of the whole system, especially when the set of states is large.

\section{Proposed guided $\mathrm{MH}$ algorithm}

To improve the $\mathrm{MH}$ selection and speed up its convergence of optimization, we propose a guided version of the MH algorithm. This guided $\mathrm{MH}$ method relies on a priori known distribution of the solution: We modify the uniform random candidate state selection to follow a predefined distribution, which is the distribution of the states that is expected to be reached at the end of the optimization. This distribution can be found by offline trials or through some learning processes.

The objective of this modification is to focus the sampling on candidates that have higher probability of being optimal than the other. After the guided selection of a candidate, this algorithm acts similarly as the original MH algorithm. The complexity of this method is similar to the one of the standard $\mathrm{MH}$ algorithm, since only one candidate state is evaluated at a time.

Note that all the three methods presented share the same complexity for the evaluation of a given local state, and also require the same exchange in information among neighboring base stations and users for evaluating the states: to determine $\mathcal{E}_{i}(s)$ for the optimization, the information to be collected and feedback should include the signal and interference powers acting on the considered node and its neighbors.

The algorithm proposed here is used for our particular resource allocation problem, but could indeed be applied to other similar optimization problems.

\section{Annealing and cooling strategies}

1) Classical logarithmic cooling: The temperature parameter $T$ can be chosen to decrease with time to perform a simulated annealing. Given a Gibbs random field, it has been proven that Boltzmann annealing, where the temperature $T$ decreases inversely proportional to the logarithm of time, is the sufficient cooling condition that guarantees the convergence of the system to global optimum as time goes to infinity [13].In brief, at a discrete time $n>1$, the temperature is defined as:

$$
T_{B}(n)=\frac{T_{0}}{\log (n)},
$$

where $T_{0}$ is the initial temperature.

The Boltzmann annealing may however be very slow to stabilize, since the temperature is decreasing in logarithmic scale. The optimization performed by simulated annealing is guaranteed for static network topology. However, it should be noted that in cellular networks, the system may vary with time, for example due to fast channel fading and/or user arrival and departure. In this paper, we assume that the system is quasi-static.

2) Fast cooling strategies: In the literature, the research on resource allocation using Gibbs sampling methods only focused on the classical logarithmic cooling strategy. We here propose to speed up the convergence, by applying faster cooling strategies:

- The "fast" annealing in [14] uses a Cauchy distribution and decreases the temperature in a linear manner:

$$
T_{C}(n)=\frac{T_{0}}{n}
$$

- The "very fast" annealing reported in [15] decreases the temperature in an exponential manner:

$$
T_{V F}(n)=\frac{T_{0}}{\exp (a n)},
$$

where $a$ is a tunable parameter.

It should be noted that there does not exist mathematical proofs that the above linear and exponential cooling strategies guarantee to converge to global optimum, under Gibbs sampling setup. Under some sampling setups (modifying the state distribution selection), the linear and exponential cooling strategies have been shown to converge to the optimal solution [15]. However, it is not clear how to cast them into distributed optimizations. Advanced studies on their implementability and convergence will be subject of future work. Here, we apply the faster cooling strategies directly into the Gibbs sampling algorithm, similarly as [16] and we have to rely on simulation results to study its performance. 


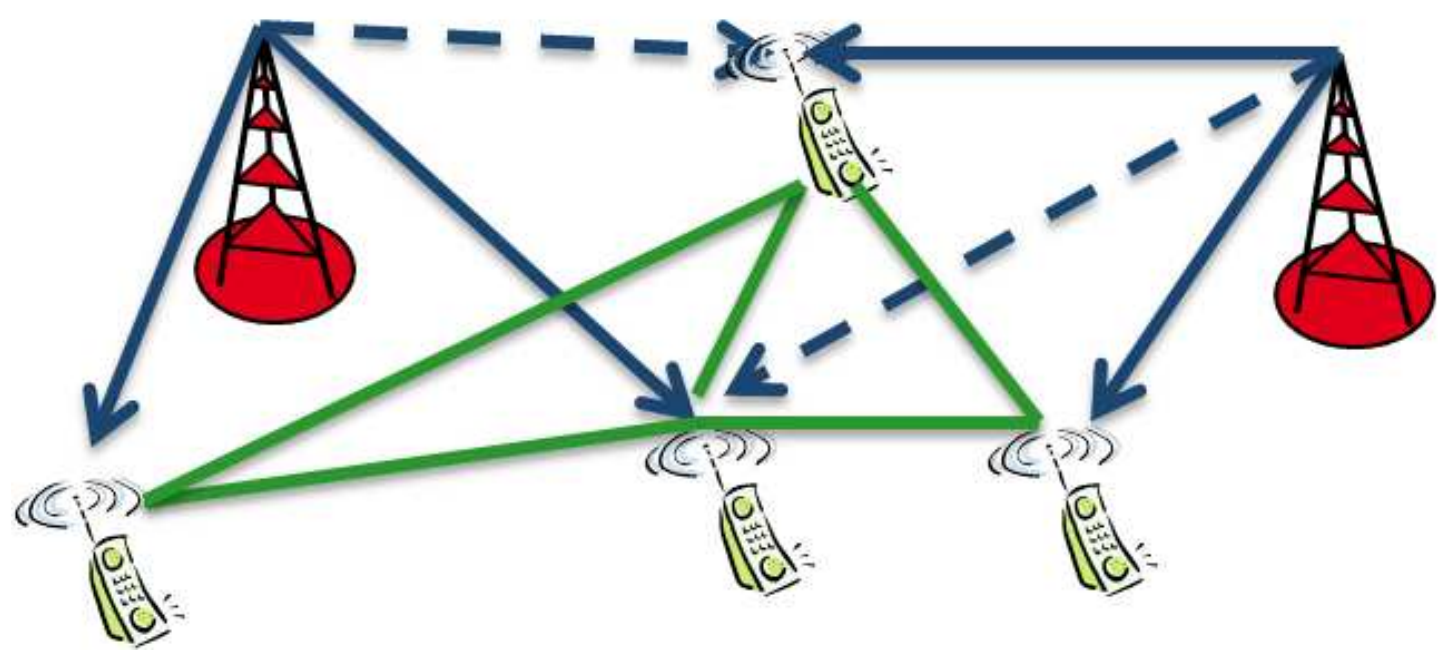

Fig. 1: An illustration of the interference graph: the set of nodes is the set of the users and the edges are represented by the green lines, transmitted signal is denoted by blue solid line, and interference is denoted by blue dashed line.

\section{APPliCATION TO THE RESOURCE AND POWER ALLOCATION PROBLEM}

\section{A. Graph of interference}

First, we define a graph to cast the problem of resource and power allocation into the Gibbs sampling framework. The graph that characterizes the system, as illustrated in Fig. 1, is defined as follows:

- The set of nodes is the set of all users $\mathcal{U}$;

- An edge $(i, j)$ is set when the transmit power of BS $b_{i}$ received at $j$ (or the power from $b_{j}$ received at $i$ ) is higher than a given threshold $\theta_{p}$. The set of neighbors of $i$ is denoted by $\mathcal{N}_{i}$;

- The state $s_{i}$ of a node $i$ is the power level on the $K$ resources assigned to it, i.e., $s_{i}=\mathbf{P}_{b_{i}}$.

\section{B. Utility function definition}

Following (9) and the development of [8], the local energy to be minimized by a node $i$ is defined by the sum of local utilities in its neighborhood:

$$
\mathcal{E}_{i}(s)=U_{i}(s)+\sum_{j \in \mathcal{N}_{i}} U_{j}(s),
$$

where $U_{i}$ is the local utility of user $i$.

In a LTE-like system, one has to perform the optimization over several parallel resources (e.g. OFDMA). As aforementioned, we focus here on a fair approach that minimizes the potential delay of all users, see (7). As shown in [10]), a representation of the global utility (7) by (8) is not trivial, due to the multiple resources and the logarithmic function (2).

In [11], it has been recently proven that in fact, we can extend the global energy to any utility function, at the price of a more information exchange among nodes: information exchange has to be performed not only among the considered node and its neighbors, but also to its neighbors' neighbors. We can therefore set the global energy to be out utility function (7) and the local energy (15) can be rewritten as:

$$
\mathcal{E}_{i}(s)=\frac{1}{C_{i}(s)}+\sum_{j \in \mathcal{N}_{i}} \frac{1}{C_{j}(s)} .
$$

We can interpret this local minimization as a trade-off between a user's utility and its neighbors' one. Increasing the transmit power of a given user to improve its data-rate will reduce its neighbors' one, due to the higher level of interference.

\section{Sequential state selection}

The Gibbs sampling requires the evaluation of the local energy for all possible states of the update node. In the present work, the allocation is performed over several resources. In our multiple resources and power allocation problem, the maximum total number of possible states for a node $i$ is $\left|\mathcal{S}_{i}\right|=L^{K}$, where $L$ is the number of power levels possible for a resource.

The cardinality of the set of local states can rapidly become impractical for a large $L$ or $K$. To overcome this issue, we choose to perform a "sequential" optimization: when a node updates its state, it will take a random permutation of all the possible resources and sequentially determine a new state considering that other resources are fixed. This leads to a maximum number of combination to be evaluated equal to $L K$, instead of taking a full size of $L^{K}$. 


\section{Simulation Results}

\section{A. Scenarios and simulation parameters}

Simulations are carried out to verify the performance of the presented methods and cooling strategies, in solving the resource and power allocation optimization problem.

The system employs a topology with 64 BS placed in classical hexagonal layout. We focus the optimization on a given chunk of $K$ resources blocks. On this chunk, a single user is allocated per station. To avoid border effects, we use a wrap-around model so that locations at the edge of the system are considered adjacent to the opposite edge. The transmit power, on each resource, can be chosen from a set including the 0 value, and a logarithmic partition of the power level from $-10 \mathrm{dBm}$ to $43 \mathrm{dBm}$, with $1 \mathrm{~dB}$ step size. Other simulation parameters are summarized in Table I.

In the simulation, one iteration corresponds to the period of time where every node has been updated sequentially but in a random ordering. Here, we assume that no two base stations will conduct a sampling exactly at the same time. This will apply generally to all the algorithms in the comparison. The results are obtained by averaging over 100 independent simulations (random user location and channel condition), each running for 200 iterations.

We aim at comparing the GS, the MH, and the guided-MH using the Boltzmann annealing (labeled as: GS-B, MH-B and guided-MH-B, resp.), as well as using the Cauchy annealing (labeled as: GS-C, MH-C and guided-MH-C, resp.) and using the exponential cooling (labeled as: GS-E, MH-E and guided-MH-E, resp.). Note that the exponential cooling is parametrized so that $T(n)=0.95^{n} T_{0}$, which is an empirical choice that fits our model [15], [16].

Additionally, the performance of a deterministic Best-Response (BR) algorithm is also shown for comparison: the BR algorithm acts similarly as the GS one, except that it always chooses the state that minimizes the local energy. This method is sub-optimal and may converge to a local minimum of the system. This local minimum is a generalized Nash equilibrium (GNE) [18].

\section{B. Initial temperature setting}

As a first step, and to efficiently use the Gibbs sampling and their variants, one should properly choose the initial temperature $T_{0}$. Except by studying the thermodynamic structure of the global system [19], there is no theoretical way to optimally determine the temperature. Fig. 2 shows the performances obtained by the GS algorithm using the logarithmic (i.e., Boltzmann: GS-B) and exponential (GS-E) cooling for some values of $T_{0}$, in comparison to the BR. Note that the results shown are particularly smooth due to the averaging over 100 independent simulations. For a single simulation, the stochastic methods progress with ups and downs before converging to a stable point value.

It is observed that when $T_{0}$ is too low (i.e., $T_{0}<0.001$ ), the randomness in the probabilistic sampling is not sufficient and the optimization acts similarly to the BR approach. On the other hand, when $T_{0}$ is too high, the convergence would take a much longer time and may not be favorable for practical usage in wireless networks, especially under the Boltzmann cooling strategy (GS-B). Besides, we can see that the different cooling strategies have different ideal initial temperature. Since the exponential cooling strategy reduces the temperature much faster than the Boltzmann one, it tends to allow higher $T_{0}$ while still converging fast enough. The result of the linear cooling strategy (GS-C) is not plotted in Fig. 2 for the readability. It follows the same trend as the exponential cooling one and its final result can be found in Fig. 3 later.

One can also see that the choice of $T_{0}$ and cooling strategies should be adapted for different scenarios, to balance convergence time and accuracy of the solution. It is still an open problem to derive optimal $T_{0}$ in analytic form. However, the present results are valid for scenarios where the variables and possible states are in a similar system setup.

\section{Cooling strategies}

We now evaluate the performance of the various sampling methods and their cooling strategies. Based on previous result and some experimental investigation, we set the initial temperature to be $T_{0}=0.001$ for the Boltzmann cooling strategy and $T_{0}=0.1$ for the Cauchy and exponential ones. These choices are motivated by the performance obtained after a reasonable number of iterations.

Fig. 3 shows the global energy utility (7), as the iterations of the optimization go on. Note that this global energy is equivalent to the sum of delays experienced by all users in the system. It is observed that the BR method converges most rapidly (in about 40 iterations). However, its final result is not optimal.

\begin{tabular}{|c|c|}
\hline Parameter & Value \\
\hline Inter-BS distance & $500 \mathrm{~m}$ \\
Number of channels $K$ & 10 \\
Bandwidth per channel & $1 \mathrm{MHz}$ \\
Noise (inc. noise figure) per channel $\sigma^{2}$ & $-105 \mathrm{dBm}$ \\
Pathloss (dB) [17], $d$ in meter & $-15.3-37.6 \log _{10}(d)$ \\
Shadowing standard dev. [17] & $10 \mathrm{~dB}$ \\
Flat-fading per resource & Rayleigh, i.i.d. \\
\hline
\end{tabular}

TABLE I: Simulation parameters 


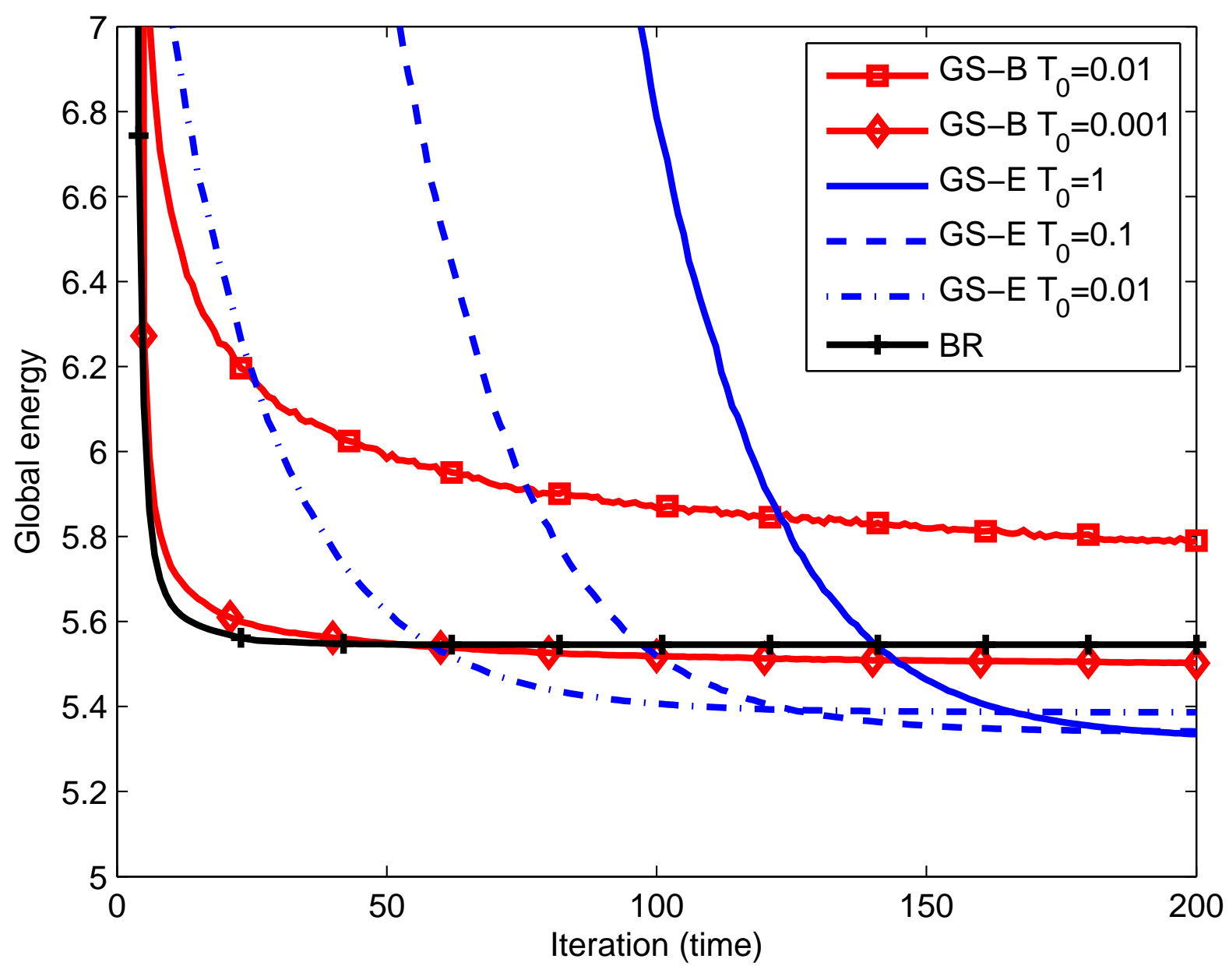

Fig. 2: Performance comparison for various initial temperatures.

In the stochastic algorithms, in particular the GS, their performances would depend on the cooling strategy. The initial temperature has been chosen so that a convergence can be reached in a finite (relatively short) time. To do so, the Boltzmann cooling strategy requires a low initial temperature and its performance is limited by the lack of randomness in the stochastic process. The final result of the GS-B strategy is thus close to the BR one but can still outperform it (but slightly). The exponential and Cauchy cooling strategies, using a higher $T_{0}$, are able to converge to better final solutions. Their performances during the initial iterations appear not favorable but soon or later outperform (in about 100 to 150 iterations). One can see that when looking at about 100 to 200 iterations, the GS-E outperforms all the other methods.

\section{Design of a guided MH algorithm}

In the simulation study, we see that the resulting OFDMA resource block and power allocation follows a particular distribution empirically as follows.

First, users use on average only $35 \%$ of their available resource blocks, and only focus on efficient channels (of low interference) to transmit data. This can be assimilated to a dynamic frequency reuse scheme to avoid high level of interference.

Second, the distribution of the power allocated to the used resource blocks follows a log-normal distribution, centered at $26 \mathrm{dBm}$ and with a standard deviation 9.5, as shown in Fig. 4. We can see a particularly high intensity at $42 \mathrm{dBm}$ due to the defined per-BS total power limitation: when a node updates its use of resource blocks, it is limited by the total power of the station (here, $43 \mathrm{dBm}$ ). In many cases, at least at least one resource block is already used with some power, so, the chosen maximum power is usually less than $43 \mathrm{dBm} .42 \mathrm{dBm}$ is therefore often a limit when some other resources are allocated with low power levels.

The distribution obtained here is system dependent, but is based on 100 independent realizations of the 64-cells hexagonal scenario. In a non-regular topology, each cell may define its state distribution according to its particular environment. We expect that the knowledge of the distribution can be obtained by the system through learning procedures or with off-line simulation 


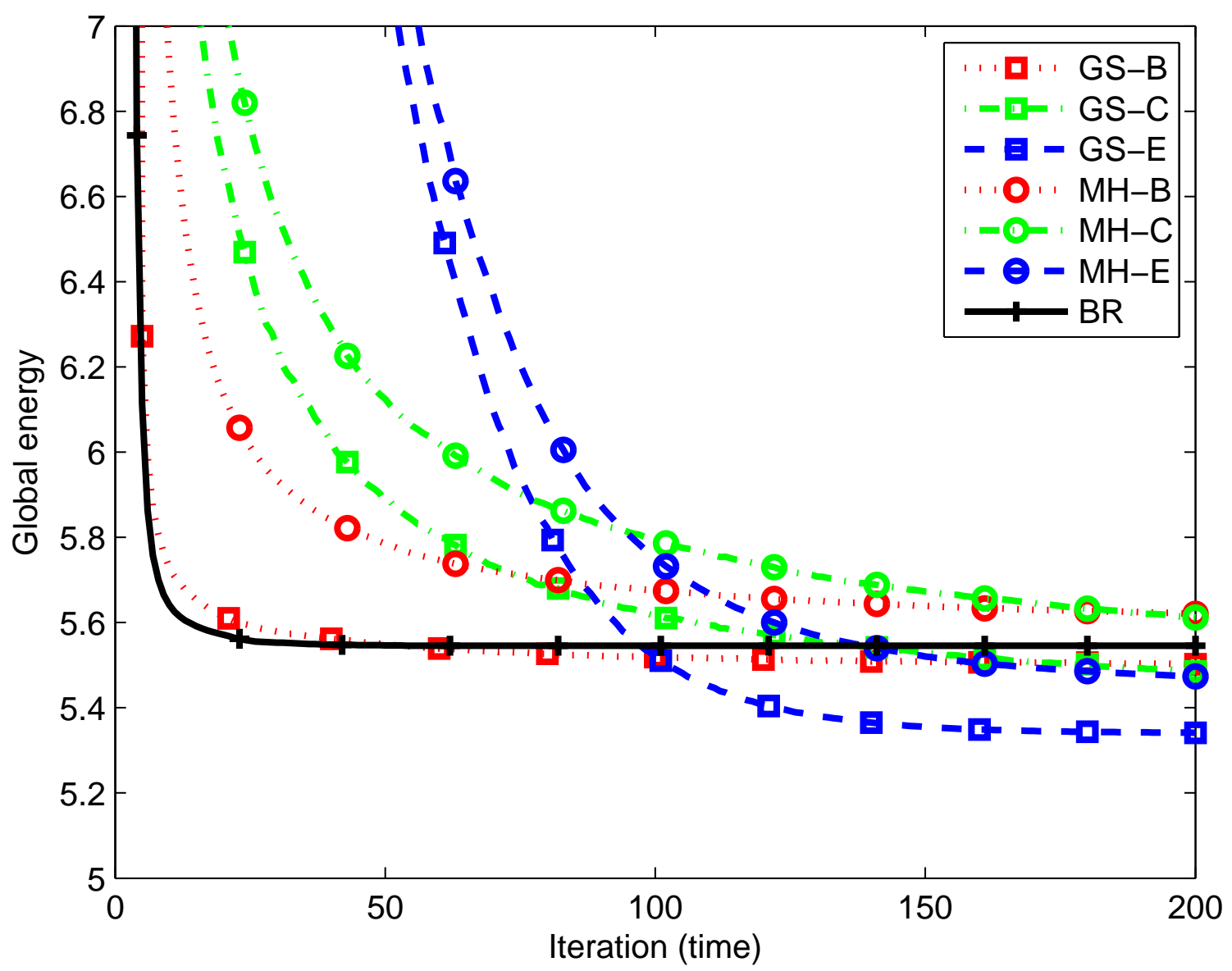

Fig. 3: Global energy of the system using different cooling strategies for the Gibbs sampling and the Metropolis-Hasting.

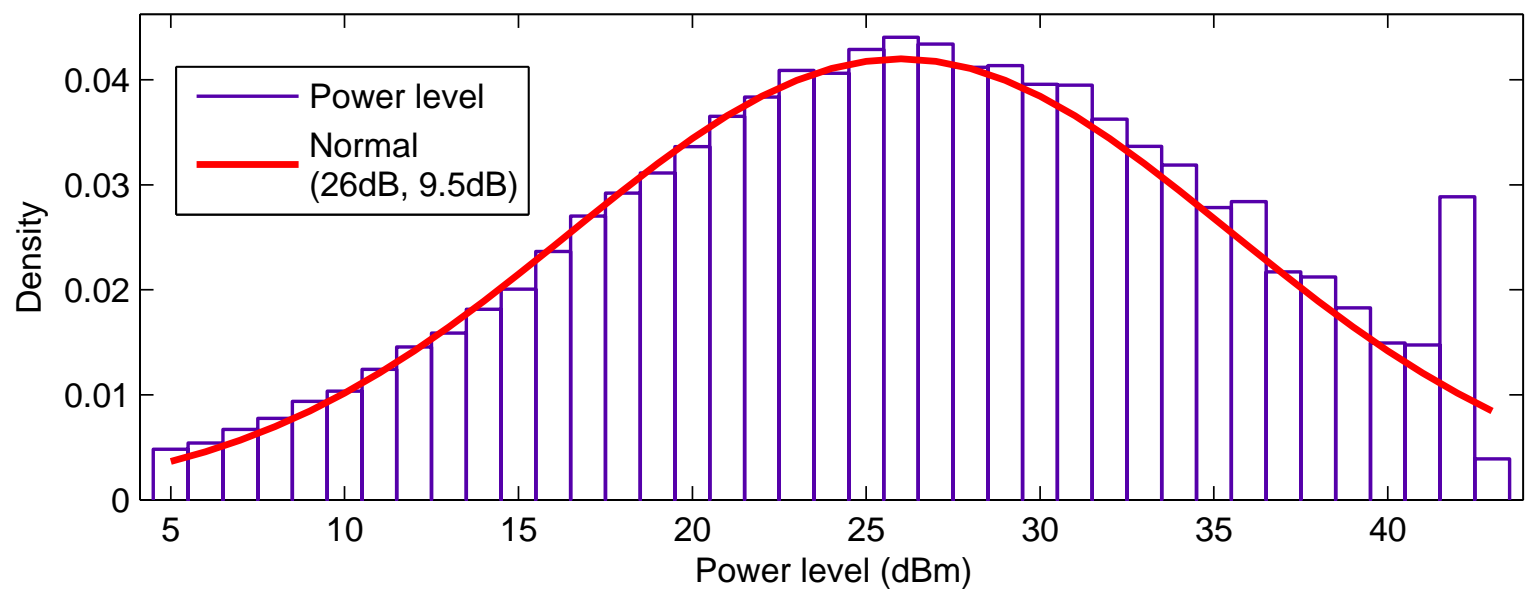

Fig. 4: Power distribution of the used resources after convergence. 


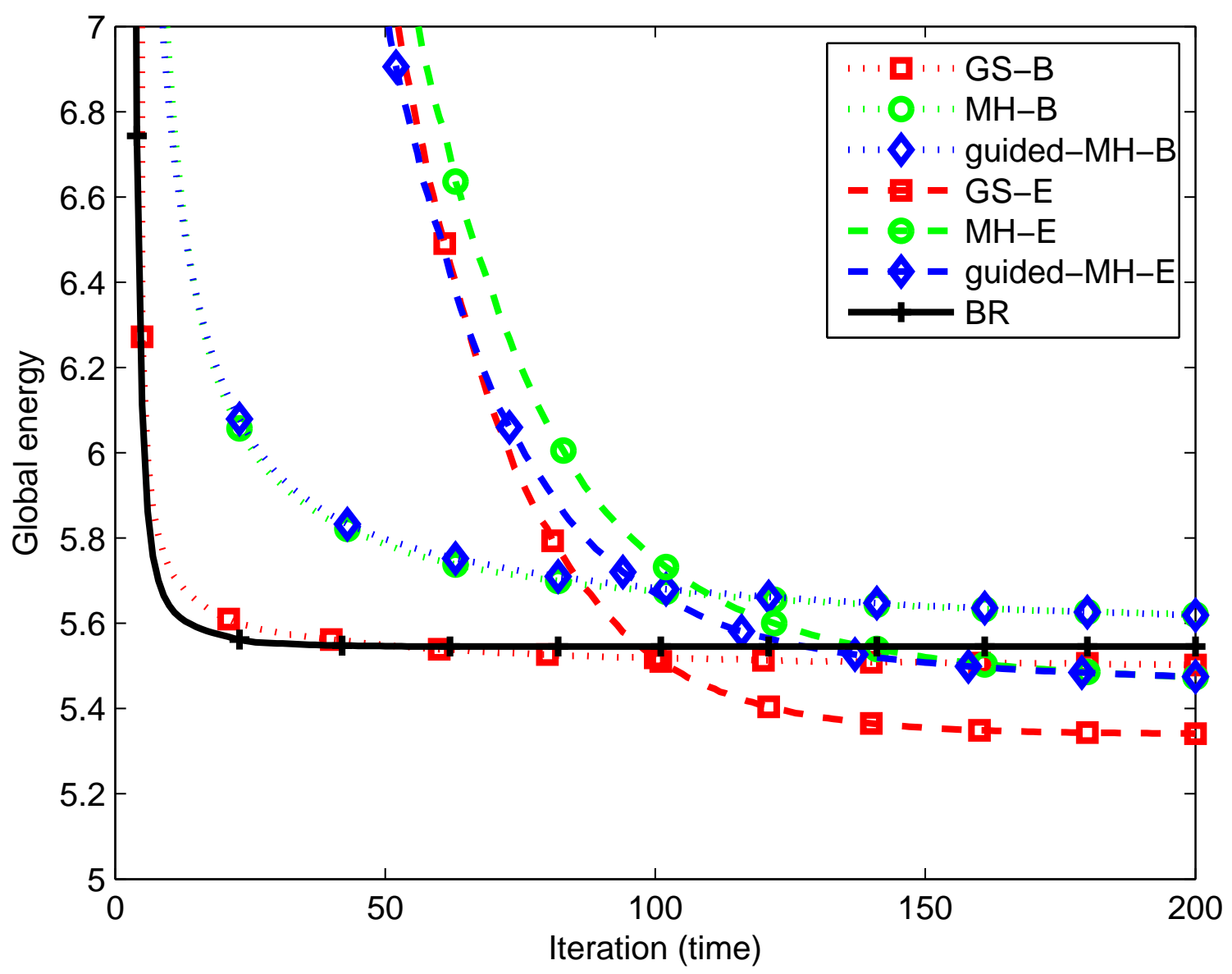

Fig. 5: Global energy of the system comparing the sampling methods for the logarithmic and exponential cooling strategies.

results. When the actual optimal distribution of each realization cannot be determined in practical system, one may use average scenario results. Note that the estimated distribution might not be the optimal choice for every realization, which can reduce the convergence speed compared to the optimal distribution.

Given the above distribution (Fig. 4), instead of using uniform distribution in $\mathrm{MH}$ sampling when choosing a candidate state, we adopt the aforementioned distribution to improve the MH sampling for its convergence and optimality. We will show the simulation result of the guided-MH scheme in next section for comparison. Notice that the following results are shown to demonstrate the effectiveness of the method, however, the principle can be applied generally to different probabilistic algorithms.

\section{E. Gibbs sampling vs. MH sampling}

We compare the performance of the different sampling methods studied in this paper. Fig. 5 shows the global energy of the system by the GS, MH and guided-MH algorithms, with the Boltzmann and exponential cooling strategies. The result of linear cooling strategy is not displayed for readability of the figure.

Comparing the above stochastic sampling methods, we see that the GS converges faster than the MH and the guided-MH. The MH and guided-MH methods may achieve similar final solution but would take a (much) longer time. On the other hand, it is observed that they still outperform the BR in a limited time (after about 140 iterations) under the exponential cooling. It is worth recalling that the computation requirement of GS is higher than that of MH due to the evaluation of a node's entire state space.

Comparing the performance of guided-MH and $\mathrm{MH}$, we see that the guided-MH which uses the predefined distribution can improve the exponential cooling scheme (guided-MH-E vs. MH-E) before a final convergence.

This is due to the fact that when the temperature of the system is high, the effect of guided sampling would be more significant since it will avoid the random sampling spend too much time on "likely not good" states. When the temperature is low (e.g., at convergence or using a low $T_{0}$ ), the $\mathrm{MH}$ and guided-MH methods show very similar performance. Recall that the 
complexity of the guided-MH is not increased compared to classical $\mathrm{MH}$, the guided sampling could be a very good option in some applications.

\section{CONCLUSION}

In this paper, we investigated the application of Gibbs sampling method and its variants for performing network optimization in a large and practical OFDMA system. We studied the impacts of sampling methods and temperature strategies on the global performance and proposed strategies of initial parameter setting. As expected, the initial temperature and cooling strategy has a fundamental role in the optimization process. Their impact could be even more important than the sampling selection method to use. Exponential cooling is the most promising cooling strategy to obtain an efficient fast optimization, while the logarithmic cooling may require a long convergence time. The $\mathrm{MH}$ provides a slower optimization than GS, due to a limited selection of the states. However, its lower complexity allows larger system and requires less information exchange in each iteration. We also proposed an improvement of the $\mathrm{MH}$ algorithm, namely guided- $\mathrm{MH}$, which can converge more rapidly than the classical $\mathrm{MH}$ algorithm. Since the guided MH algorithm has a low computation cost, it could be considered as an alternative of cost-effective method.

\section{ACKNOWLEDGEMENT}

This work has been partially funded by the Beijing Natural Science Foundation Major Project (4110001), the National Science and Technology Major Project (2012ZX03003005-004) and the 2010 Main Direction Program of Knowledge Innovation of Chinese Academy of Sciences. The research leading to these results has received funding from the European Union's Seventh Framework Programme (FP7/2007-2013) under grant agreement no. 318115. Part of the work presented in this paper has been carried out at LINCS (www.lincs.fr).

\section{REFERENCES}

[1] Small Cell Forum, "Small cells - what's the big idea," White Paper, Feb. 2012.

[2] S. Koskie and Z. Gajic, "Signal-to-interference-based power control for wireless networks: A survey, 1992-2005," Dynamics of Continuous, Discrete and Impulsive Systems B: Applications and Algorithms, vol. 13, no. 2, pp. 187-220, 2006.

[3] C. S. Chen, K. W. Shum, and C. W. Sung, "Round-robin power control for the weighted sum rate maximisation of wireless networks over multiple interfering links," European Transactions on Telecommunications, vol. 22, no. 8, pp. 458-470, 2011.

[4] M. J. Nawrocki, M. Dohler, and A. H. Aghvami, Understanding UMTS Radio Network Modelling, Planning and Automated Optimisation: Theory and Practice. Wiley, 2006.

[5] E. Altman, T. Boulogne, R. El-Azouzi, T. Jiménez, and L. Wynter, "A survey on networking games in telecommunications," Computers \& Operations Research, vol. 33, no. 2, pp. 286-311, 2006.

[6] S. Geman and D. Geman, "Stochastic relaxation, Gibbs distributions, and the Bayesian restoration of images," IEEE Trans. on Pattern Analysis and Machine Intelligence, , vol. PAMI-6, no. 6, pp. 721-741, Nov. 1984.

[7] N. Metropolis, A. Rosenbluth, M. Rosenbluth, A. Teller, and E. Teller, "Equation of state calculations by fast computing machines," J. Chem. Phys., vol. 21, no. 6, pp. 1087-1092, 1953.

[8] B. Kauffmann, F. Baccelli, A. Chaintreau, V. Mhatre, K. Papagiannaki, and C. Diot, "Measurement-based self organization of interfering 802.11 wireless access networks," in IEEE Infocom, 2007, pp. 1451-1459.

[9] C. S. Chen and F. Baccelli, "Self-optimization in mobile cellular networks: Power control and user association," in IEEE ICC, 2010.

[10] V. Garcia, C. S. Chen, N. Lebedev, and J.-M. Gorce, "Self-optimized precoding and power control in cellular networks," in IEEE PIMRC, 2011.

[11] S. C. Borst, M. G. Markakis, and I. Saniee, "Non-concave utility maximization in locally coupled systems with applications to wireless and wireline networks," IEEE Trans. on Networking (TON), 2013.

[12] L. Massoulié and J. Roberts, "Bandwidth sharing: objectives and algorithms," IEEE/ACM Trans. Netw., vol. 10, no. 3, pp. 320-328, 2002.

[13] P. Brémaud, Markov Chains: Gibbs Fields, Monte Carlo Simulation, and Queues. Springer Verlag, 1999.

[14] H. H. Szu and R. L. Hartley, "Nonconvex optimization by fast simulated annealing," Proceedings of the IEEE, vol. 75, no. 11, pp. 1538-1540, 1987.

[15] L. Ingber, "Very fast simulated re-annealing," Math. Comput. Modelling, vol. 12, pp. 967-973, 1989.

[16] P. Arora, N. Xia, and R. Zheng, "A Gibbs sampler approach for optimal distributed monitoring of multi-channel wireless networks," in IEEE Globecom, Dec. 2011, pp. 1-6.

[17] 3GPP TR 25.996, "Spatial channel model for multiple input multiple output (MIMO) simulations," 3GPP Tech. Rep. v10.0.0, Dec. 2009.

[18] F. Facchinei and C. Kanzow, "Generalized Nash equilibrium problems," A Quarterly Journal of Operations Research, vol. 5, pp. 173-210, 2007.

[19] B. Andresen, K. H. Hoffmann, K. Mosegaard, J. Nulton, J. M. Pedersen, and P. Salamon, "On lumped models for thermodynamic properties of simulated annealing problems," J. Phys., vol. 49, pp. 1485-1492, 1988. 